Combined capillary force and step and flash lithography

This content has been downloaded from IOPscience. Please scroll down to see the full text. 2005 Nanotechnology 16391

(http://iopscience.iop.org/0957-4484/16/4/010)

View the table of contents for this issue, or go to the journal homepage for more

Download details:

IP Address: 216.165.126.139

This content was downloaded on 08/09/2015 at 03:15

Please note that terms and conditions apply. 


\title{
Combined capillary force and step and flash lithography
}

\author{
Elisa Mele, Francesca Di Benedetto, Luana Persano, \\ Dario Pisignano and Roberto Cingolani \\ Department of Engineering of Innovation, National Nanotechnology Laboratory of Istituto \\ Nazionale di Fisica della Materia, University of Lecce, Via Arnesano I-73100 Lecce, Italy \\ E-mail: elisa.mele@unile.it
}

Received 14 September 2004, in final form 5 January 2005

Published 7 February 2005

Online at stacks.iop.org/Nano/16/391

\begin{abstract}
The combination of a key element of soft lithography, namely the elastomeric stamp, with the operation principle of step and flash imprint lithography results in a moulding procedure allowing high throughput and remarkable operational simplicity. $100 \mathrm{~nm}$ scale dense features can be fabricated via in situ polymerization of a polyurethane fluid, simultaneous to the capillary penetration into the recessed regions of high-resolution elastomeric elements. Excellent pattern definition has been obtained for features down to $200 \mathrm{~nm}$, with aspect ratio of around unity over areas of the order of $\mathrm{cm}^{2}$. The physical principles of the fluidic motion within the sub- $\mu \mathrm{m}$ channels are also discussed, to estimate the maximum aspect ratio achievable before the complete curing of the employed photopolymer.
\end{abstract}

\section{Introduction}

Photolithography has so far been the dominant technology for patterning surfaces with resolution in the sub- $\mu \mathrm{m}$ scale. Over the past decade, extensive efforts have been made to develop alternative patterning methods, such as lithography by electron and ion beams, x-rays, scanning probes [1], soft [2] and imprinting [3,4] lithographies, self-assembling [5], and atom lithography [6].

In particular, soft lithography [2] comprises a set of techniques (microcontact printing [5], replica moulding [7], microtransfer moulding [8], micromoulding in capillaries [9], solvent-assisted micromoulding [10] and so on), which rely on the use of an elastomeric stamp or replica to transfer a pattern onto a substrate. The replica is straightforwardly prepared by casting the liquid prepolymer of an elastomer against a master with a patterned relief structure on its surface. In this way, multiple copies of the highly complex structures in the master can be faithfully produced with nanometre resolution.

Other effective mechanical lithographies, such as nanoimprinting [4] and step and flash imprint lithography (SFIL), employ hard masters, from which the pattern is transferred onto the target organic compounds by applying a pressure. In particular, SFIL is a high-throughput method to generate $100 \mathrm{~nm}$ and sub-100 nm scale patterns via in situ curing of suitable polymers under pressure [11, 12].
This technique makes use of a low-viscosity, photo-curable liquid, which allows one to carry out the imprint process at room temperature with low applied pressures, and by a transparent, rigid template, in order to obtain a complete exposure of the photopolymer. The complete release of the imprint mould from the imprinted polymer generally requires proper anti-sticking treatments of the template surface [13]. This drawback can be overcome by the use of a poly(dimethylsiloxane) (PDMS) stamp. In fact, PDMS is chemically inert, hence it does not adhere irreversibly to the surface of the target polymers, and it can easily be released after photopolymerization. Moreover, the elastic characteristics of PDMS allow the replica to spontaneously wet the surface and make conformal contact with the substrate, without applying external forces, over a large area (up to many $\mathrm{cm}^{2}$ ).

In this paper, we report our recent results concerning the effective patterning approach resulting from the combination of the key element of soft lithography, namely the use of the elastomeric stamp, with the operation principle of step and flash imprint lithography (SFIL). Suitable composite stamps are employed to pattern a photopolymer by the capillary effect, driving it into the void spaces of the features formed between the replica and the film, with high resolution (up to $200 \mathrm{~nm}$ ) and aspect ratio (up to unity). This presents the advantages of both capillary force and step and flash lithography, allowing 


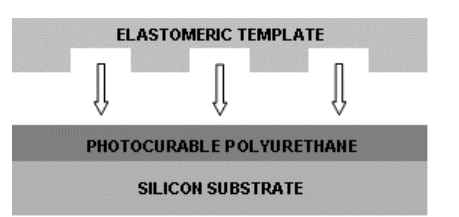

Contact between the elastomeric replica and UV-curable film

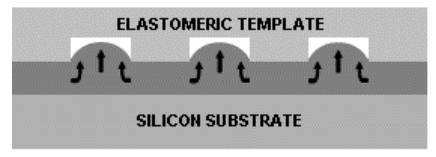

Filling of the void spaces of the features formed between the replica and the photo-curable film by capillary effects during polymerization

Template separation after the polymerization

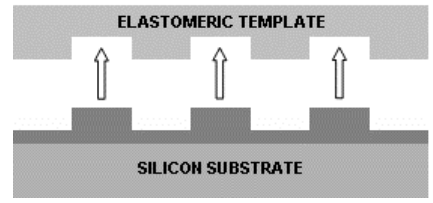

Figure 1. Schematic diagram of the capillary-combined SFIL process by elastomeric replicas (features not in scale).

highly parallel and fast operation, easy alignment procedures by the transparent mould, and chemical flexibility.

\section{Experimental details}

First, we fabricated our master structures consisting of squares with lateral dimensions varying from 20 to $2 \mu \mathrm{m}$, parallel grooves of period 600 and $400 \mathrm{~nm}$, and duty-cycle $50 \%$. Master patterns with squares were realized onto silicon by photolithography, whereas master patterns with parallel grooves were fabricated by electron beam lithography (EBL) by a Raith Elphy Plus pattern generator controlling a Leica Stereoscan 440 scanning electron microscope (SEM), and subsequent reactive ion etching by means of a $\mathrm{CF}_{4} / \mathrm{Ar}$ mixture properly chosen in order to minimize the plasmainduced polymer deposition. Details concerning the etching parameters are reported elsewhere [14].

A scheme of the capillary-combined SFIL process is shown in figure 1. We fabricated the elastomeric replicas of the masters by PDMS (Sylgard 184, Dow Corning, Midland, MI). The Sylgard 184 base (A) and curing agent (B) are mixed in a ratio of nine parts $\mathrm{A}$ and one part $\mathrm{B}$, in weight. The silicone elastomer was cast on the master and polymerized in situ at $140{ }^{\circ} \mathrm{C}$ for $15 \mathrm{~min}$, according to a standard replica moulding procedure [2]. For high-resolution soft lithography, composite stamps of hard PDMS ( $h$-PDMS) and PDMS [15] were also realized. The $h$-PDMS solution was prepared mixing $3.4 \mathrm{~g}$ of trimethylsiloxy-terminated vinylmethylsiloxanedimethylsiloxane (VDT-731, ABCR, Karlsruhe, Germany), $18 \mu \mathrm{l}$ of a Pt catalyst (platinum divinyltetramethyldisiloxane, SIP6831.1, ABCR, Karlsruhe, Germany), and $5 \mu \mathrm{l}$ of a modulator (2,4,6,8-tetramethyltetravinylcyclotetrasiloxane, Sigma Aldrich, St Louis, MO). Afterwards the mixture was degassed for 1-2 min, $1 \mathrm{~g}$ of methylhydrosiloxanedimethylsiloxane (HMS-301, ABCR, Karlsruhe, Germany)
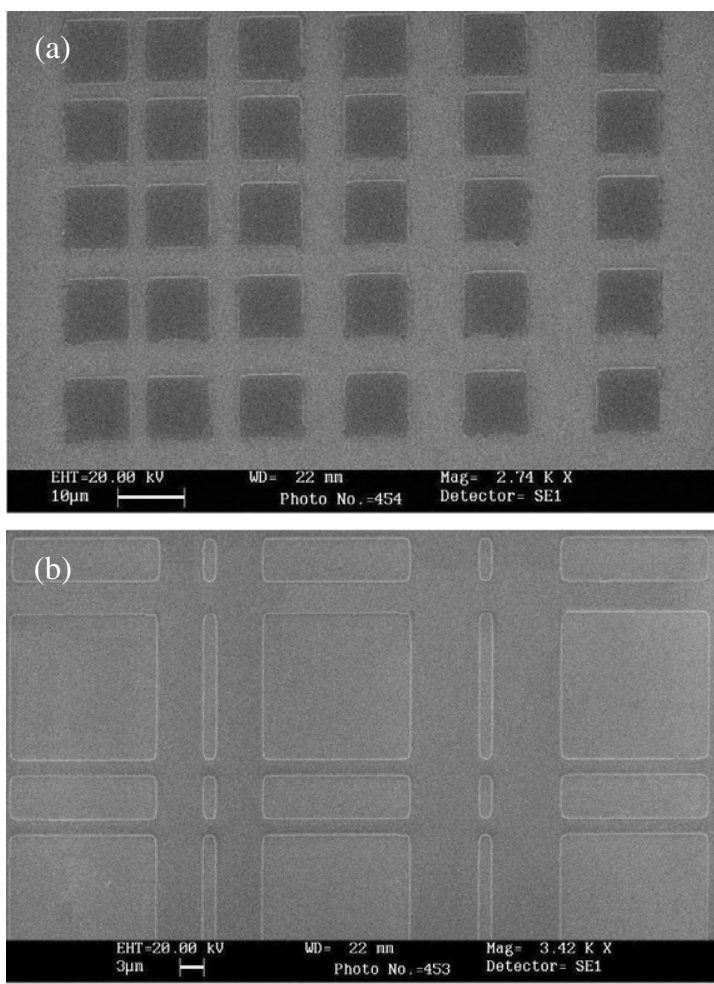

Figure 2. 1-10 $\mu \mathrm{m}$-scale patterns realized by SFIL with the PDMS replicas: squares with $10 \mu \mathrm{m}$ side at variable mutual distance (a), and squares of $20 \mu \mathrm{m}$ side alternating with rectangles with lateral dimensions varying between 2 and $20 \mu \mathrm{m}$ (b).

was added, and the $h$-PDMS solution was gently stirred. We then spin-coated (1500 rpm for $40 \mathrm{~s}$ ) a thin $h$-PDMS layer onto the master surface, and cured it at $60^{\circ} \mathrm{C}$ for $30 \mathrm{~min}$. After the polymerization, Sylgard 184 PDMS, in the form of liquid pre-polymer, was poured onto the $h$-PDMS layer and cured at $60{ }^{\circ} \mathrm{C}$ for $1 \mathrm{~h}$. Then, the composite stamp was carefully peeled off from the master surface.

The elastomeric replicas were then used to reproduce the master patterns into films of low viscosity, photopolymerizable polyurethane (NOA 72, Norland Optical Adhesive, NJ), spincoated at $2000 \mathrm{rpm}$ for $40 \mathrm{~s}$ onto various substrates, including silicon, metals, and glass. In the soft lithographic procedure, the stamp is placed on the substrate, without preliminary surface treatments, to let the NOA 72 fill the grooves of the mould by capillary force. Immediately after positioning the replica (i.e. during the capillary penetration) the system is exposed to UV light through the backside of the template, thereby cross-linking NOA 72 at room temperature. After the polymerization of the polyurethane film, the mould is separated from the substrate, and the pattern remains on the target. The elastic nature and the chemical inertia of the PDMS and $h$ PDMS allowed us to obtain an easy and complete release of the template from the polyurethane layer and permit the use of the same replica for several imprint processes.

\section{Results and discussion}

The plan-views of patterns of different geometry, realized by Sylgard 184 PDMS replicas, are shown in figure 2: both squares of $10 \mu \mathrm{m}$ side placed at variable distances 
Combined capillary force and step and flash lithography
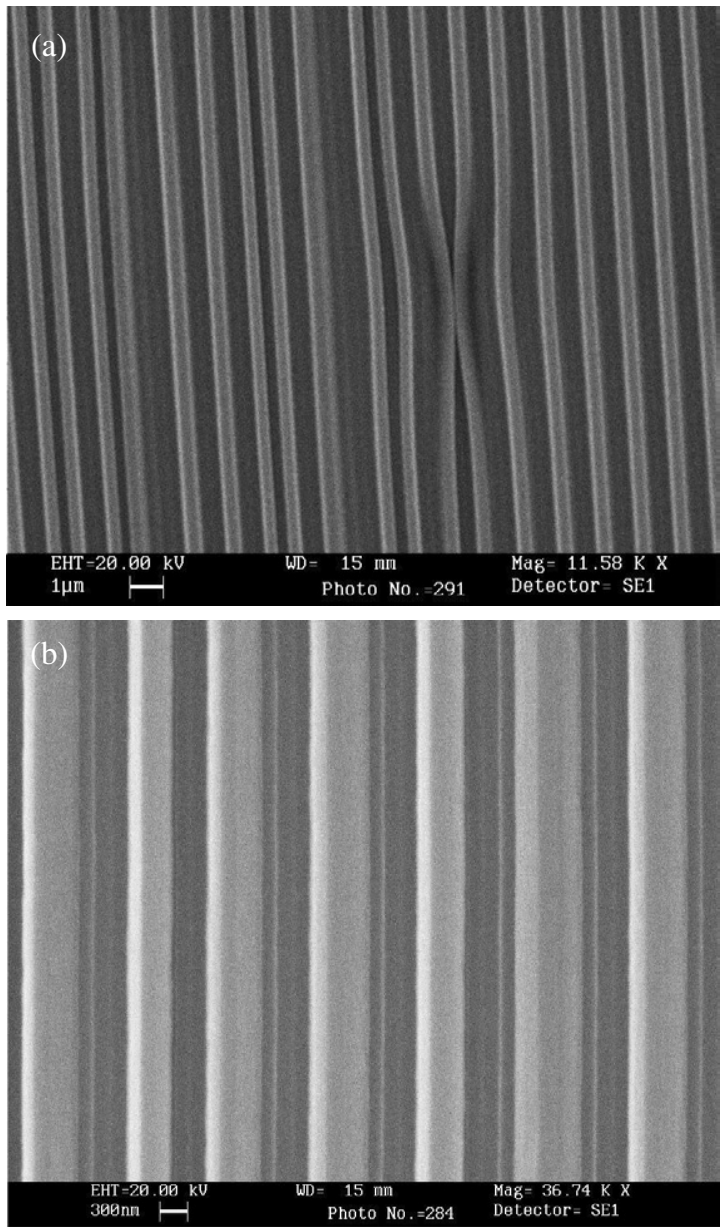

Figure 3. Polyurethane imprinted patterns realized by capillary-combined SFIL with standard (Sylgard 184) PDMS replicas for (a) a $600 \mathrm{~nm}$ period and (b) a $400 \mathrm{~nm}$ period grating.

(figure 2(a)) and squares of $20 \mu \mathrm{m}$ side alternating with rectangles of 2-20 $\mu \mathrm{m}$ lateral dimensions (figure 2(b)) present well defined features. We underline that the capillary-based SFIL technique offers a number of advantages over other imprinting methods, such as the possibility of avoiding high temperature and pressure during patterning, and the easier template alignment, because of the use of a low viscosity photopolymer and of a transparent stamp, respectively. In addition, the use of elastomeric templates allows us to avoid the anti-sticking surface treatment of the mould, which is otherwise necessary to obtain a complete release of the mould from the substrate in the other printing lithographies. The replica moulding prolongs the life of the original master, avoiding the stresses due to the direct use in the imprinting step. Finally, whereas the conventional SFIL and nanoimprint lithographies transfer a negative copy of the master in the target compounds, this approach enables positive-tone lithography on photopolymers, since the imprinted patterns reproduce the same shape (positive) of the starting masters by the use of elastomeric replicas.

In our experiments, Sylgard 184 replicas were also employed for transferring patterns with features in the sub- $\mu \mathrm{m}$ range. However, in this case we observed a poor imprinting quality, revealed by the presence of defects along the periodic nanostructures, often presenting a period different from that

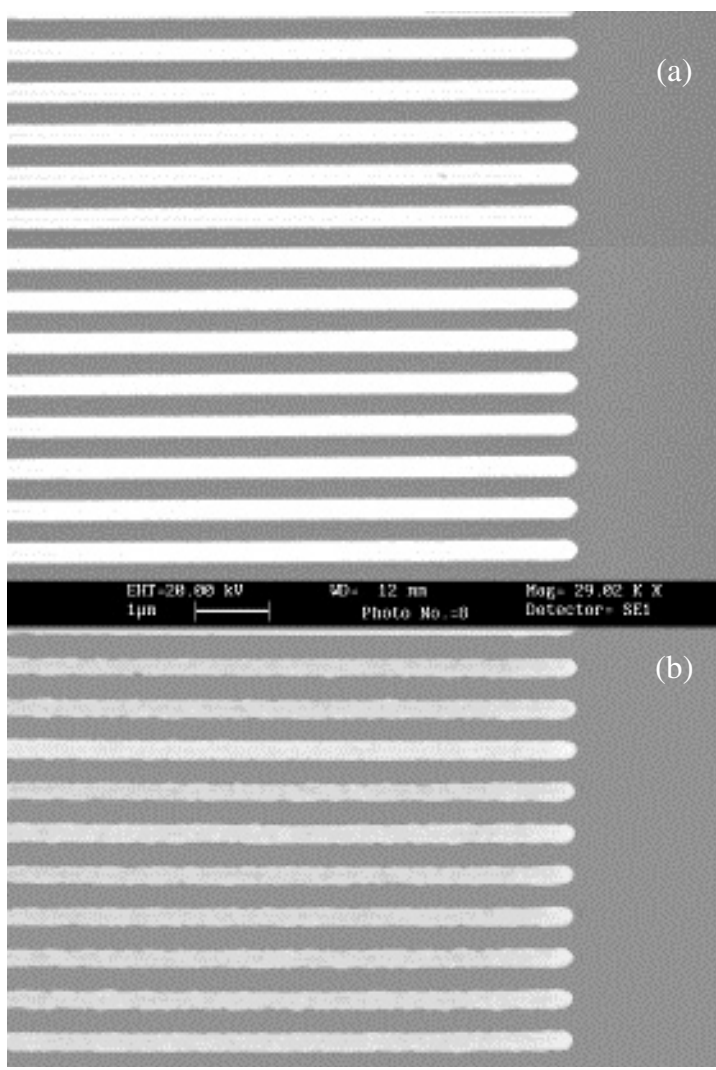

Figure 4. (a) Master of a $600 \mathrm{~nm}$ period grating, and (b) corresponding imprinted pattern realized by capillary-combined SFIL with a PDMS/ $h$-PDMS composite mould.

of the master. In fact, though the mechanical stability of PDMS nanostructures can be sufficient for certain applications, including microring [16] and distributed feedback optical resonators [17], it is well established that its low Young modulus (3 $\mathrm{MPa}$ ) can determine the collapse and the pairing of high-aspect-ratio elastomeric features. These effects are demonstrated by the polyurethane imprinted patterns shown in figure 3, displaying a $600 \mathrm{~nm}$ period (figure 3 (a)), and a $400 \mathrm{~nm}$ period grating (figure 3 (b)), in which many defects are visible in terms of periodicity ((a) and (b)) and of geometry (such as the crossing structure in (a)), due to distortions of the elastomeric features and to pairing and uncompleted conformal contact, respectively.

In order to push the limit of resolution of the patterning approach down to the $100 \mathrm{~nm}$ scale, the capillary-combined SFIL process by EBL-fabricated masters was carried out by composite moulds employing the $h$-PDMS, which is characterized by a large Young modulus (9 $\mathrm{MPa}$ ) [15-18].

Also for capillary-combined SFIL, the accurate generation of sub- $500 \mathrm{~nm}$ patterns could be obtained by $h$-PDMS. Figure 4 compares a $600 \mathrm{~nm}$ period master grating and the pattern imprinted by a composite mould, demonstrating the excellent fidelity achieved with the replication procedure. These features were imprinted by us with the same fidelity onto areas up to the order of $\mathrm{cm}^{2}$. Moreover, we realized well defined features with $200 \mathrm{~nm}$ lateral dimension (figure 5), and depth of (197 \pm 5) $\mathrm{nm}$, determining an aspect ratio of about unity. The very good pattern definition indicates that the achieved resolution 

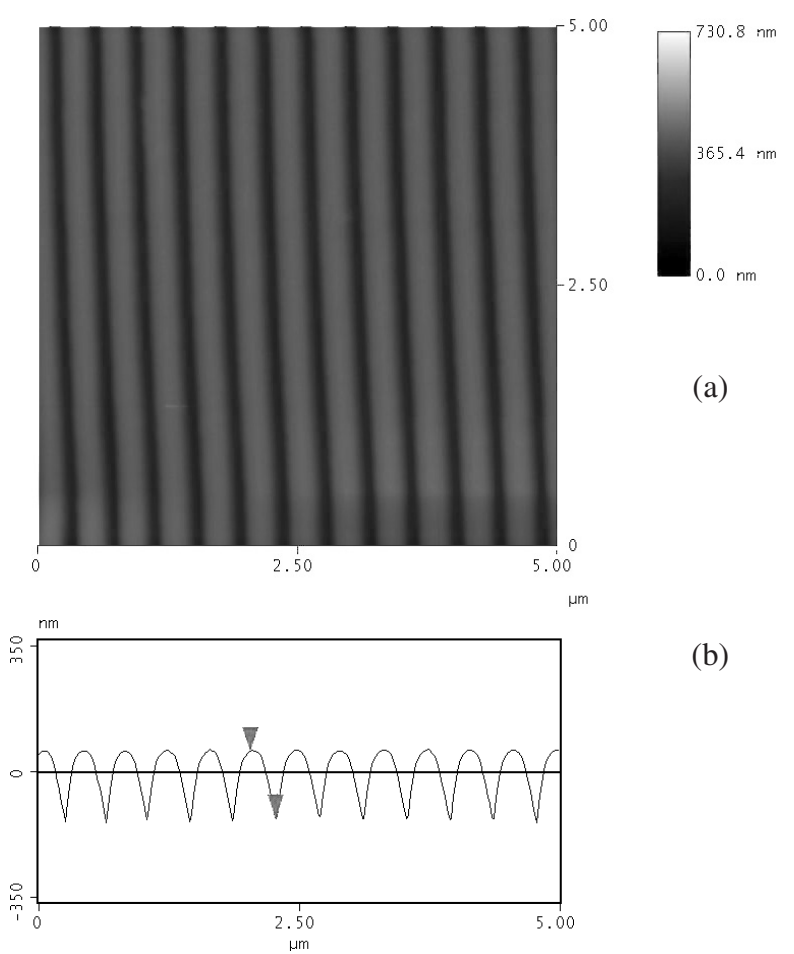

Figure 5. (a) Two-dimensional view, imaged by AFM, and (b) corresponding cross section of a polyurethane imprinted $400 \mathrm{~nm}$ grating.

depends on the starting masters. We expect that capillarycombined SFIL at sub-100 $\mathrm{nm}$ scale will be certainly reliable, by the use of $h$-PDMS composite stamps. Soft lithography methods with elastomeric moulds [19] and nanoimprinting techniques [3] have been demonstrated for the replication of features of the order of $10 \mathrm{~nm}$, and capillary-combined SFIL should be able to reach the same level of resolution.

In the capillary-combined SFIL process, the pattern transfer is due to the penetration of the polymer into the recessed features of the elastomeric mould by capillarity. This is strongly favoured, especially at the early stage of the process, by the low viscosity of the employed UV-curable polymer. In fact, the filling rate of the fluidic channels depends on the viscosity of the polymer, $\eta$, according to the equation [20]

$$
\frac{\mathrm{d} z}{\mathrm{~d} t}=\frac{R \gamma \cos \Theta}{4 \eta z},
$$

where $R$ is the hydraulic radius of the capillary, $\gamma$ is the surface tension at the polymer/air interface $\left(40 \mathrm{mN} \mathrm{m}^{-1}\right.$ [21]), $\Theta$ is the contact angle between the polymer and the surface of the capillary (around $80^{\circ} \mathrm{C}[22]$ ), and $z$ is the vertical coordinate indicating the penetration into the recessed feature. Neglecting the effect of gravity and considering a constant value of the polymer viscosity, the filled height as a function of the time, $t$, needed for the penetration process can be expressed as follows:

$$
z=\left(\frac{R \gamma t \cos \Theta}{2 \eta}\right)^{1 / 2} .
$$

However, since in the capillary-combined SFIL the photopolymer is cured simultaneous to the capillary penetration, its viscosity varies during the filling of channels as a function of the curing time. Assuming a polymerization kinetics determining an exponential increase of the polyurethane viscosity [23],

$$
\eta(t)=\eta_{0} \exp \left(k_{0} t\right)
$$

where $\eta_{0}$ is the initial viscosity and $k_{0}$ is the viscosity-rate kinetic constant, equation (2) becomes

$$
z=\left(\frac{R \gamma \cos \Theta}{2 \eta_{0} k_{0}}\left(1-\exp \left(-k_{0} t\right)\right)\right)^{1 / 2}
$$

The maximum height that the polymer can reach by capillarity during the polymerization before becoming solid can therefore be estimated straightforwardly. For instance, by NOA 72 polyurethane with initial viscosity of $155 \times 10^{-3} \mathrm{~Pa} \mathrm{~s}$ and a viscosity-rate kinetic constant of $8.2 \times 10^{-3} \mathrm{~s}^{-1}$ (this value was obtained by the observed time needed for the polymer to go from the initial value of the viscosity to a solidlike form), a maximum feature height of about $2.3 \mathrm{~mm}$ is estimated for the periodic grooves of radius of $1 \mu \mathrm{m}$, within the time necessary for a complete curing (of the order of $1 \mathrm{~h}$ ). Equation (4) provides an overestimated value for the achievable aspect ratio, because it describes a homogeneous photocuring reaction, which is often not the case for UVinitiated polymer curing. In addition, our model neglects the effect of gravity, which tends to counteract the vertical capillary penetration. However, the estimated value indicates that the obtainable depth of the polymeric imprinted patterns could be larger than that demonstrated here, and that features of higher aspect ratio could be realized by the fabrication of suitable master structures.

\section{Conclusion}

We demonstrated and elucidated the physical principles of an imprinting technique that is a combination of capillary force and step and flash imprint lithography. The advantages offered by capillary-combined SFIL in terms of high resolution and throughput can be further improved by the employment of composite PDMS/ $h$-PDMS templates. Varying from procedures described very recently for other photo-polymerisable compounds [24], the capillary-induced filling of the elastomeric channels does not require any applied pressure. The excellent quality of the $100 \mathrm{~nm}$ scale patterns, transferred onto the target polymer by the conformal contact and the subsequent, simultaneous curing and capillary penetration, clearly suggests this approach as a candidate for promising nanofabrication methods on polymers.

\section{References}

[1] Ross C B, Sun L and Crooks R M 1993 Langmuir 9632

[2] Xia Y and Whitesides G M 1998 Angew. Chem. Int. Edn Engl. 37550

[3] Chou S Y, Krauss P R and Renstrom P J 1995 Appl. Phys. Lett. 673114

[4] Chou S Y, Krauss P R and Renstrom P J 1996 Science 27285

[5] Kumar A and Whitesides G M 1993 Appl. Phys. Lett. 632002

[6] Mutzel M, Tandler S, Haubrich D, Meschede D, Peithmann K, Flaspohler M and Buse K 2002 Phys. Rev. Lett. 88083601

[7] Xia Y, Kim E, Zhao X-M, Rogers J A, Prentiss M and Whitesides G M 1996 Science 273347 
[8] Zhao X-M, Xia Y and Whitesides G M 1996 Adv. Mater. 8837

[9] Kim E, Xia Y and Whitesides G M 1995 Nature 376581

[10] Kim E, Xia Y, Zhao X-M and Whitesides G M 1997 Adv. Mater. 9651

[11] Colburn M et al 1999 Proc. SPIE: Emerg. Lithogr. Technol. $\mathbf{3 6 7 6} 379$

[12] Colburn M, Grot A, Amistoso M, Choi B J, Bailey T, Ekerdt J, Sreenivasan S V, Hollenhorst J and Willson C G 2000 Proc. SPIE: Emerg. Lithogr. Technol. 3997453

[13] Bailey T, Choi B J, Colburn M, Meissl M, Shaya S, Ekerdt J G, Sreenivasan S V and Wilson C G 2000 J. Vac Sci. Technol. B 183572

[14] Pisignano D, Persano L, Gigli G, Visconti P, Stomeo T, De Vittorio M, Barbarella G, Favaretto L and Cingolani R 2004 Nanotechnology 15766

[15] Schmid H and Michel B 2000 Macromolecules 333042

[16] Huang Y, Paloczi G T, Scheuer J and Yariv A 2003 Opt. Express 112452
[17] Pisignano P, Mele E, Persano L, Gigli G, Visconti P, Cingolani R, Barbarella G and Favaretto L 2004 Phys. Rev B 70205206

[18] Odom T W, Love J C, Wolfe D B, Paul K E and Whitesides G M 2002 Langmuir 185314

[19] Xia Y, McClelland J J, Gupta R, Qin D, Zhao X-M, Sohn L L, Celotta R J and Whitesides G M 1997 Adv. Mater 9147

[20] Mayers D 1999 Surface, Interface, and Colloids (New York: Wiley-VCH) pp 97-118

[21] Krupenkin T N, Taylor J A, Shneider T M and Yang S 2004 Langmuir 203824

[22] Pisignano D, Gigli G, Visconti P, Zocco A, Perrone A and Cingolani R 2002 J. Vac. Sci. Technol. B 202248

[23] Malkin A Y, Kulichikhin S G, Kerber M L, Gorbunova I Y and Murashova E A 1997 Polym. Eng. Sci. 371322

[24] Choi W M and Park O Ok 2004 Nanotechnology 15135 